\title{
Swim or chill: lifestyles of a bacillus
}

\author{
David Dubnau ${ }^{1}$ \\ Public Health Research Center, New Jersey Medical School, Newark, New Jersey 07103, USA
}

Bacillus subtilis exhibits a number of lifestyles, each characterized by a distinguishing pattern of gene expression. In this issue of Genes \& Development, Chai and colleagues (pp. 754-765) dissect the decision-making processes underlying the switch between the motile and sessile states of $\boldsymbol{B}$. subtilis, and show a role for a doublenegative feedback loop that locks the system into the sessile state. These findings are discussed in the context of the natural history and of other lifestyle decisions faced by this model organism.

Bacteria-Bacillus subtilis in particular-continue to fascinate researchers. This "simple" organism resides in the region of soil surrounding plant roots, called the rhizophere, where it forms multicellular, organized biofilms (Earl et al. 2008). B. subtilis exhibits a number of additional lifestyle adaptations that are subject to regulation by a network of signal transduction proteins. Best known is its ability to differentiate into resistant spores, and, accordingly, sporulation has long been the subject of intense scrutiny in many laboratories as a model system for the study of bacterial development. After sporulation, the most investigated aspect of the $B$. subtilis lifestyle is competence, a physiological state in which a bacterial cell stops dividing and expresses a set of genes for the internalization of environmental DNA, eventually incorporating this DNA into its own genome in a process known as transformation. A hallmark of competence is its bimodal expression. Only a minority of cells in a competent population expresses the DNA transport genes. Individual cells therefore exist in two distinct states with respect to competence gene expression: ON and OFF.

More recently, it has become apparent that natural isolates of $B$. subtilis have other talents that are not fully recapitulated in domesticated laboratory strains (Earl et al. 2008). These tricks were neglected despite decades of familiarity, because in some respects the laboratory derivatives of wild and free $B$. subtilis are tame versions of their natural cousins. For example, domesticated laboratory strains have lost the ability to form robust biofilms, and, in fact, produce wimpy structures compared with their probable ancestor, strain 3610. From

[Keywords: Epigenetic; bistability; biofilm; Bacillus subtilis]

${ }^{1}$ Correspondence.

E-MAIL dubnauda@umdnj.edu; FAX (973) 854-3453.

Article is online at http://www.genesdev.org/cgi/doi/10.1101/gad.1923110. work on both laboratory strains and a natural isolate, it is known that the SinR repressor protein inhibits the transcription of important biofilm operons (eps and yqxM), and has therefore been considered the master regulator of biofilm development (Kearns et al. 2005). The SinI protein directly antagonizes SinR activity (Bai et al. 1993), and because only free $\operatorname{Sin} R$ acts as a repressor of these operons, the synthesis of SinI is an important early step in biofilm formation. As in competence, SinI is produced in a small subpopulation of cells, and consequently only these cells express the biofilm genes (Chai et al. 2008).

In this issue of Genes \& Development, Chai et al. (2010) clarify another lifestyle. Dividing cells of $B$. subtilis come in two flavors-swimming and sessile-and these cell types coexist in the same population. Sessile daughter cells remain attached to one another following cell division because they fail to produce autolytic enzymes needed to degrade the peptidoglycan cell wall material between the cells, and they are nonmotile because they do not make flagella. The genes that encode the flagellar and autolytic enzymes are OFF in the sessile cells and ON in the motile ones-another case of a binary switch, like that of competence. Clearly, genotype is not everything; clonal populations can adopt alternative lifestyles, and, remarkably, these can coexist in a uniform environment. Again, the domesticated strain differs from strain 3610 in its motile-sessile switch behavior; it is biased toward the sessile lifestyle during growth, whereas the wild strain exhibits the opposite bias.

Intuition suggests that stochastic processes must lie at the heart of the decision that selects each of these lifestyles: to swim or to chill, to form biofilms or to remain pelagic, and to become competent or not. Random processes in biology have received much attention recently, and are important for a wide variety of important decision-making events (Raj and van Oudenaarden 2008). Indeed, experimental evidence shows that the competence decision depends on random cell-to-cell variation (noise) in the basal (uninduced) expression of $\operatorname{comK}$, a key regulatory gene (Suel et al. 2006; Maamar et al. 2007). The product of this gene, ComK, drives the transcription of DNA uptake genes as well as of its own gene, establishing a simple, positive autoregulatory loop. The binding of ComK to its own promoter appears to be cooperative, and this, together with the loop, results in two-state behavior. Once the switch is thrown, a cell is locked in the ON state by the resulting high, self-reinforcing level of ComK. 
Chai et al. (2010) have now explored the regulatory network that governs the sessile-motile choice. In an elegant series of genetic and biochemical experiments, they track down and make sense of what must have been, initially, puzzling phenotypes. The results are very satisfying, and, as in the case of competence, positive autoregulation is central, although the circuitry is more involved (see Fig. 1 in Chai et al. 2010). Chai et al. (2010) have discovered that $\operatorname{SinR}$ is a repressor of another regulatory gene, slrR, and that the SlrR protein in turn sequesters SinR, preventing it from repressing the biofilm operons. Instead of the simple autoregulation of ComK, a double-negative feedback loop lies at the heart of the motile-sessile decision. What happens when sinI transcription is activated, due, for example, to a random burst in its basal rate of expression? In that particular cell, SinI will be synthesized and will bind to SinR, antagonizing its ability to repress slrR. The resulting elevated level of SlrR protein will then further inhibit SinR, reinforcing the effect of SinI synthesis and locking the cell in a lowSinR, high-SlrR state. Remarkably, the SlrR-SinR complex then binds to the promoters of autolysin and, presumably, flagellar genes as well, inhibiting expression and thus preventing daughter cell separation and motility. During growth, random cells in which sinI expression remains low will exist in a state with active SinR and a low concentration of SlrR, and daughter cells will consequently separate and go their merry ways.

But there is more to this marvelous story. Presumably, the stochastic switching just described has evolved as a bet-hedging strategy. Some cells become motile and swim off to make their fortunes, whereas others stay close to their sisters, adopting other means of ensuring the perpetuation and reproduction of the shared genome. Generally, in wild populations, the relevant noise levels and binding affinities must be under selective pressure to set optimal rates of transition between ON and OFF states. In the competence system, a programmed uptick in the average basal expression of $\operatorname{comK}$ occurs as cells enter stationary phase, followed closely by a decrease in this expression. This transient uptick brings the average closer to the threshold for transition to competence, temporarily increasing the rate of transition, and then abruptly closes the window of opportunity for the development of competence (Maamar et al. 2007). The uptick is dependent on Spo0A P (N Mirouze and D Dubnau, unpubl.), but not on ComK itself. Thus, a deterministic mechanism modulates an inherently stochastic decision by adjusting the threshold, thereby regulating the transition rate to competence as cells cease growing.

Chai et al. (2010) also suggest that the sessile-motile switch can operate in a deterministic mode in developing cells. As B. subtilis cultures enter the stationary phase, the response regulator Spo0A is phosphorylated gradually, and its target promoters respond in a temporal sequence that depends on their binding affinities for Spo0A P. The promoter of $\sin I$ is one of the first to respond, switching cells to the sessile lifestyle. Although it is known that only $1 \%-2 \%$ of the population exhibits elevated expression of $\sin I$, the factors that determine this bimodal expression are not known. Because the expression of $\sin I$ is bimodal, it is likely that a stochastic choice mechanism still operates. In my opinion, this is not a purely deterministic mechanism, but, as with competence, it is a "mixed" stochastic decision in which the probability of transition is modulated by a programmed (deterministic) mechanism. Strikingly, both the motility and competence systems depend on SpoOA for this behavior. The biological rationale for restricting $\operatorname{sinI}$ expression to only a few cells appears to be that this expression inactivates $\operatorname{Sin} \mathrm{R}$ and therefore derepresses the yqxM and eps operons in these same cells. These operons encode products that manufacture an extracellular protein/polysaccharide matrix in which all of the cells in a developing biofilm are embedded. The randomly chosen few therefore serve the many, at least initially-an example of cell specialization within a bacterial "tissue." Perhaps the remaining cells not only conserve energy by not producing matrix unnecessarily, because the chosen few make enough, but they are also free to explore other avenues for survival, including motility, competence, and spore formation.

As always, as our understanding deepens, new questions arise: (1) As already noted, the domesticated strains of $B$. subtilis are biased toward the sessile lifestyle, probably because a steady diet of boiled cow extract has made them lazy and they have mutated in the laboratory to conserve the energy that would otherwise be expended on swimming. Their country cousins, on the other hand, are biased toward the motile lifestyle. Although this sessile bias in the domesticated strain is due to a mutation in the swr gene, what is the role and mechanism of action of the Swr protein? (2) As Chai et al. (2010) point out, SinI is needed for the switch to a sessile lifestyle during growth, because a sinI loss-of-function mutant is locked permanently in the motile state. But still unclear are the factors that lead to noise in $\sin I$ expression. Is this intrinsic noise, caused by cell-to-cell variation in the firing rate of the sinI promoter? Or is the noise extrinsic, due to variation in the activities of upstream genes? For example, the phosphorylated form of the master regulator Spo0A directly activates the sinI promoter. Perhaps the random cells with elevated SinI also have higher than average amounts of SpoOA P. (3) What are the relative affinities of SinI and SlrR for SinR, how is SinR partitioned between SinI and SlrR, and how much free SinR is present? Modeling the switch circuitry will be aided greatly by knowledge of these values. (4) Another potentially interesting and unexplored aspect of the system is its reversibility. For example, how do sessile cells in biofilms become motile? Is there a mechanism to degrade one of the key players, permitting escape from the sessile state?

A more general question concerns the roles of the various lifestyle choices in the natural history of the organism. How has a soil bacterium evolved to do all of these things, involving intricate and delicately poised decision-making mechanisms? What portentous factors operate in the real world to necessitate such lifestyle choices? Perhaps free B. subtilis exist in the soil mostly as 
inactive spores (Earl et al. 2008). Real life, according to this view, may begin when a spore encounters a plant root. We may envisage the outlines of a natural history of B. subtilis. Perhaps root contact triggers a succession of events: spore germination, growth, motility, chemotaxis, switch to the sessile state, competence and biofilm formation, motility, escape and spread to another site, and, eventually, sporulation. These events may unfold in a preferred sequence, similar to the one just suggested. However, an element of stochastic choice may serve to hedge the bets at several of these steps. In this way, a population of cells with identical genomes retains the flexibility to adapt quickly to changing circumstances. For now, the sessile-motile switch is a beautiful example of bacterial decision-making. In the future, it is up to the community of scientists now studying $B$. subtilis to move from flasks and Petri dishes to models of the real world. Only in that way will we get to the "root" of the matter.

\section{Acknowledgments}

Thanks to members of my laboratory for discussions, to Nico Mirouze for unpublished results, and to Rich Losick for comments on the manuscript. Work mentioned from my laboratory was supported by NIH grants GM57720 and GM043756.

\section{References}

Bai U, Mandic-Mulec I, Smith I. 1993. SinI modulates the activity of SinR, a developmental switch protein of Bacillus subtilis, by protein-protein interaction. Genes \& Dev 7: 139148.

Chai Y, Chu F, Kolter R, Losick R. 2008. Bistability and biofilm formation in Bacillus subtilis. Mol Microbiol 67: 254-263.

Chai Y, Norman T, Kolter R, Losick R. 2010. An epigenetic switch governing daughter cell separation in Bacillus subtilis. Genes \& Dev (this issue). doi: 10.1101/gad.1915010.

Earl AM, Losick R, Kolter R. 2008. Ecology and genomics of Bacillus subtilis. Trends Microbiol 16: 269-275.

Kearns DB, Chu F, Branda SS, Kolter R, Losick R. 2005. A master regulator for biofilm formation by Bacillus subtilis. Mol Microbiol 55: 739-749.

Maamar H, Raj A, Dubnau D. 2007. Noise in gene expression determines cell fate in Bacillus subtilis. Science 317: 526529.

Raj A, van Oudenaarden A. 2008. Nature, nurture, or chance: Stochastic gene expression and its consequences. Cell 135: 216-226.

Suel GM, Garcia-Ojalvo J, Liberman LM, Elowitz MB. 2006. An excitable gene regulatory circuit induces transient cellular differentiation. Nature 440: 545-550. 


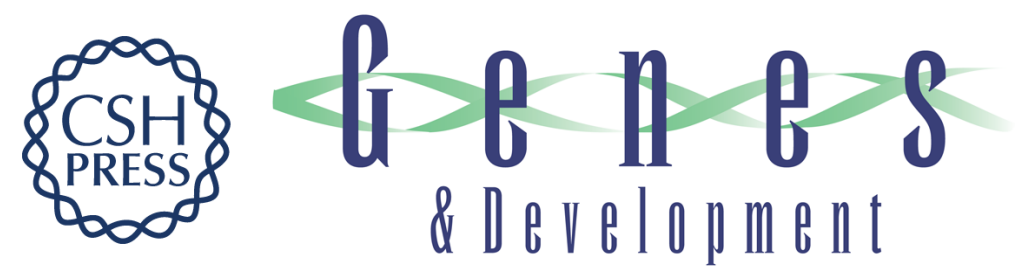

\section{Swim or chill: lifestyles of a bacillus}

David Dubnau

Genes Dev. 2010, 24:

Access the most recent version at doi:10.1101/gad.1923110

Related Content An epigenetic switch governing daughter cell separation in Bacillus subtilis Yunrong Chai, Thomas Norman, Roberto Kolter, et al. Genes Dev. April , 2010 24: 754-765

References This article cites 8 articles, 3 of which can be accessed free at: http://genesdev.cshlp.org/content/24/8/735.full.html\#ref-list-1

Articles cited in:

http://genesdev.cshlp.org/content/24/8/735.full.htm|\#related-urls

\section{License}

Email Alerting

Receive free email alerts when new articles cite this article - sign up in the box at the top Service right corner of the article or click here.

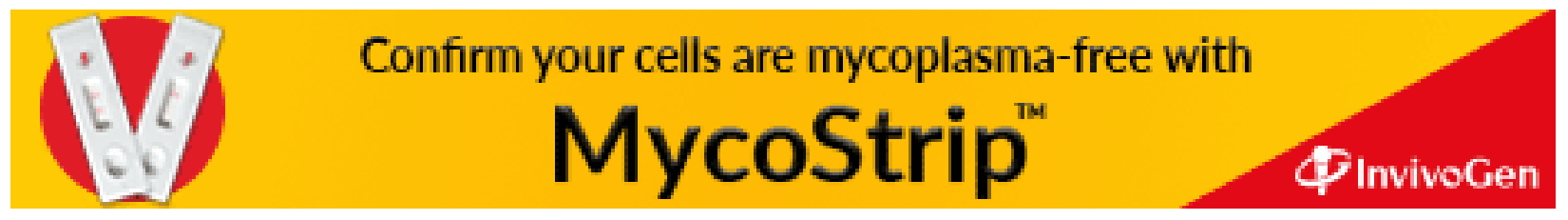

